\title{
Images of the month 2: Disseminated gonococcal infection presenting as the arthritis-dermatitis syndrome
}

\author{
Authors: Chi-Hui Wang ${ }^{A}$ and Chun-Wei Lu ${ }^{B}$
}
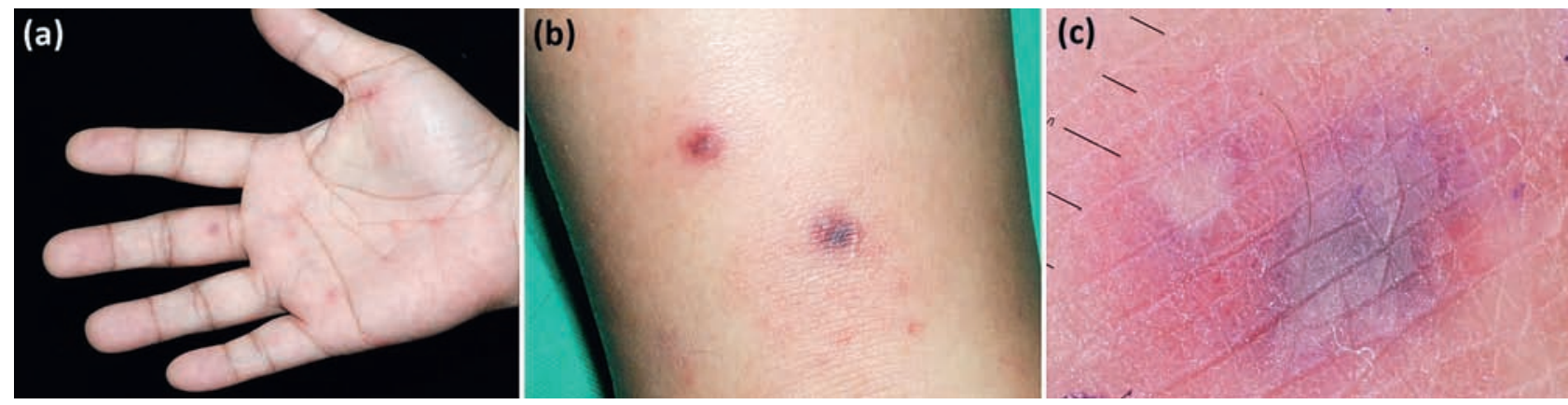

Fig 1. Disseminated gonococcal infection. (a) Discrete, tender, erythematous macules and vesicles on the right palm and middle finger. (b) Right calf with haemorrhagic rash and pustules formation. (c) Dermoscopic image showing the purpuric macule with dusky centre and peripheral erythema.

\section{Case}

A 47-year-old man presented with multiple tender, erythematous-to-violaceous macules and pustules on his palms and lower legs (Fig 1). Spike fever, malaise and arthralgia in wrists and right ankle were noted for 3 days. There was no history of urogenital symptoms. Laboratory surveillance showed leucocytosis of $17.3 \times 10^{3} / \mu \mathrm{L}$ and increased C-reactive protein of $30.74 \mathrm{mg} / \mathrm{dL}$. Skin biopsy demonstrated leucocytoclastic vasculitis. Remarkably, blood culture was positive for Gramnegative diplococci, subsequently identified as Neisseria gonorrhoeae. Under a focused history-taking concerning sexually transmitted infections (STIs), the patient reported having had unsafe sexual behaviours with female prostitutes 2 weeks ago. Screening tests of syphilis, chlamydia and HIV were all negative. On commencement of intravenous ceftriaxone, $2 \mathrm{~g}$, and oral azithromycin, $1 \mathrm{~g}$, daily for disseminated gonococcal infection (DGI), he became afebrile with marked resolution of skin rash and polyarthralgia 6 days later.

\section{Discussion}

The incidence of DGI has been reported to be very low $0.5-3 \%$ of all gonococcal infections). ${ }^{1}$ Historically, DGI occurred more

Authors: Adermatology resident, Chang Gung Memorial Hospital,

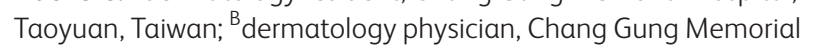
Hospital and Chang Gung University, Taoyuan, Taiwan frequently in woman with occult cervical infections; however, rising cases were observed among HIV-infected males recently. DGI results from the bloodstream dissemination of $N$ gonorrhoeae, typically developing within 3 weeks of primary mucosal infection.

Clinical suspicion of DGI should arise in patients with a classic triad of skin rash, polyarthralgia and tenosynovitis, termed 'arthritisdermatitis syndrome. ${ }^{2}$ Characteristic cutaneous lesions are scattered small-sized purpuric macules on palms and soles, which can evolve into vesicopustules with hemorrhage. ${ }^{3}$ Histologically, epidermal and perivascular neutrophilic infiltrates with vasculitis may be seen. Asymmetric polyarticular pain and tenosynovitis involving small joints are distinctive features. DGI is mainly confirmed by culturing gonococci from blood, synovial fluid or skin lesion. Cultures of synovial fluid and skin lesion are mostly negative, but positive blood cultures are identified in $50 \%$ of patients with arthritis-dermatitis syndrome of DGI. Differential diagnosis includes infective endocarditis, meningococcemia and Reiter syndrome.

With the rapid emergence of antimicrobial-resistant N gonorrhoeae, the Centers for Disease Control and Prevention recommends dual antibiotic therapy (ceftriaxone plus azithromycin) for DGI. ${ }^{4}$ To date, STIs including gonorrhoeal infection are growing global health concerns. Timely identification of DGI and initiation of appropriate treatment are mandatory to prevent potential complications and transmission. -

\section{References}

1 Beatrous SV, Grisoli SB, Riahi RR, Matherne RJ, Matherne RJ. Cutaneous manifestations of disseminated gonococcemia. Dermatol Online J 2017;23. 
2 Brogadir SP, Schimmer BM, Myers AR. Spectrum of the gonococcal arthritis-dermatitis syndrome. Semin Arthritis Rheum 1979;8:177-83.

3 Ghosn SH, Kibbi A-G. Cutaneous gonococcal infections. Clin Dermatol 2004;22:476-80

4 Workowski KA, Bolan GA. Sexually transmitted diseases treatment guidelines, 2015. Morbidity and mortality weekly report:

Recommendations and reports 2015;64:1-137.
Address for correspondence: Dr Chun-Wei Lu, Department of Dermatology, Chang Gung Memorial Hospital, Linkou Branch, 5 Fushin Street, Taoyuan, Taiwan.

Email: c.wei.lu@gmail.com 